\title{
THE RATLIFF-RUSH IDEALS IN A NOETHERIAN RING
}

\author{
William Heinzer, David Lantz, and Kishor Shah \\ Purdue University, Colgate University, \\ and Southwest Missouri State University
}

Introduction. Let $R$ be a Noetherian ring and $I$ be a regular ideal in $R$. (By ring we mean a commutative ring with unity, and by a regular ideal we mean one that contains a nonzerodivisor.) Then the ideals of the form $I^{n+1}:_{R} I^{n}=\left\{x \in R \mid x I^{n} \subseteq I^{n+1}\right\}$ increase with $n$. The union of this family is an interesting ideal first studied by Ratliff and Rush in [RR]. Let us denote:

$$
\widetilde{I}=\bigcup\left\{I^{n+1}:_{R} I^{n} \mid n \geq 1\right\}
$$

Ratliff and Rush show in particular that $\widetilde{I}$ is the largest ideal for which, for sufficiently large positive integers $n,(\widetilde{I})^{n}=I^{n}$ and hence that $\widetilde{\widetilde{I}}=\widetilde{I}$. We call regular ideals $I$ for which $I=\widetilde{I}$ Ratliff-Rush ideals, and we call $\widetilde{I}$ the Ratliff-Rush ideal associated with I. It is easy to see that an element $a$ of $I^{n+1}: I^{n}$ is integral over $I$, in the sense that there is an equation of the form $a^{k}+b_{1} a^{k-1}+\ldots+b_{k}=0$, where $b_{i} \in I^{i}$ for $i=1, \ldots, k$. Therefore, the ideal $\widetilde{I}$ is always between $I$ and the integral closure $I^{\prime}$ of $I$, and hence integrally closed ideals are Ratliff-Rush ideals. Ratliff and Rush observe [RR, (2.3.4)] that the powers of an invertible ideal are Ratliff-Rush ideals, so any principal ideal generated by a nonzerodivisor is a Ratliff-Rush ideal. They also prove the interesting fact that for any regular ideal $I$ of $R$, there is a positive integer $n$ such that for all $k \geq n, \widetilde{I^{k}}=I^{k}[\mathrm{RR},(2.3 .2)]$, i.e., all sufficiently high powers of a regular ideal are Ratliff-Rush.

A regular ideal $I$ is always a reduction of its associated Ratliff-Rush ideal $\widetilde{I}$, in the sense that $I(\widetilde{I})^{n}=(\widetilde{I})^{n+1}$ for some positive integer $n$. For the basic facts on reductions and reduction numbers of ideals, we refer the reader to [NR], [H1], and [H2]. In particular, if there is an element $a$ of an ideal $I$ for which $a I^{n}=I^{n+1}$ then $a R$ is called a principal reduction of $I$ and the smallest $n$ for which this equation holds is called the reduction number of $I$. We will call a regular ideal $I$ stable iff it has a principal reduction with reduction number at most one, i.e., iff there is an element $a$ of $I$ for which 
$a I=I^{2}$. (For a regular ideal in a one-dimensional local ring, this definition is equivalent to that of Lipman [L, Definition 1.3 and Corollary 1.11].)

In Section 1 we discuss some general properties of Ratliff-Rush ideals, consider the behavior of the Ratliff-Rush property with respect to certain ideal and ring-theoretic operations, and try to indicate how one might determine whether or not a given ideal is Ratliff-Rush. Section 2 centers around the fact that the associated Ratliff-Rush ideal $\widetilde{I}$ of a nonzero ideal $I$ in a Noetherian domain $R$ can be realized as the contraction to $R$ of the extension of $I$ to an appropriate extension domain of $R$. This is especially useful in the case where $R$ is one-dimensional. We illustrate with a number of examples involving one-dimensional local domains. We show in Theorem 2.8 that every Ratliff-Rush ideal of a one-dimensional local domain $R$ is either principal or integrally closed iff there are no rings properly between $R$ and the integral closure of $R$. In [RR, (2.5)], a one-dimensional local domain in which every ideal is either principal or integrally closed provides an example where every nonzero ideal is Ratliff-Rush. In Example 2.10 (ii), we give examples where every Ratliff-Rush ideal is either principal or integrally closed, but there are nonzero ideals that are not Ratliff-Rush.

Motivated by another comment of Ratliff and Rush [RR, (2.5)], we classify in Section 3 the Noetherian domains in which every nonzero ideal is Ratliff-Rush. It is easily seen that a Noetherian domain with this property is of dimension at most one, and that the condition is preserved under localization. We show in Theorem 3.9 that a one-dimensional local domain $R$ with integral closure $R^{\prime}$ has the property that every nonzero ideal in $R$ is Ratliff-Rush iff every nonzero ideal in $R$ is stable, and that these conditions are almost equivalent to the following: for every $x \in R^{\prime}$, the dimension of $R[x] / M R[x]$ over $R / M$ is at most two, and for every $x, y \in R^{\prime}$, the dimension of $R[x, y] / M R[x, y]$ over $R / M$ is at most three. If $R^{\prime}$ is a finitely generated $R$-module, then every nonzero ideal in $R$ is Ratliff-Rush is equivalent to $R$ having multiplicity at most two, but an example of Sally and Vasconcelos in [SV2, Example 5.4, page 335] shows that there exists a one-dimensional local domain of multiplicity three in which every nonzero ideal is stable. It follows from Theorem 3.3 of $[\mathrm{R}]$ that a one-dimensional local domain $(R, M)$ of multiplicity greater than two in which every nonzero ideal is Ratliff-Rush satisfies a number of stringent conditions, which are recalled and supplemented in Corollary 3.11 below.

Following the suggestion of Bass [B1, page 324], we use for examples 
and illustrations complete one-dimensional local domains of the form $R=$ $k\left[\left[t^{s}: s \in S\right]\right]$ consisting of formal power series in the indeterminate $t$ with coefficients from a field $k$ and exponents from an additive submonoid $S$ of the nonnegative integers that contains all sufficiently large integers; and we consider ideals in $R$ generated by monomials $t^{n}$. The minimal number of generators of the maximal ideal of such an $R$ is the minimal number of generators of the monoid $S$. The multiplicity of $R$ is the smallest positive integer in $S$. The integral closure of $R$ is $k[[t]]$, so the integrally closed ideals in $R$ consist of all elements in $R$ with order (= degree of the smallest-degree nonzero term) greater than or equal to some integer. Any ideal $I$ in $R$ has a principal reduction; if $I$ is generated by monomials, then one such reduction is $t^{n} R$ where $n$ is the smallest order among the nonzero elements of $I$ (also called the order of the ideal $I$ ).

In the present paper, the rings under consideration will usually be integral domains, so that all nonzero ideals are regular. Our use of the term local ring includes the Noetherian hypothesis. We use ' to denote integral closure of a ring in its total quotient ring or integral closure of an ideal within its ring (not within the total quotient ring).

\section{General properties of Ratliff-Rush ideals.}

(1.1) If $I$ is a stable ideal in a Noetherian ring $R$, then $I$ is Ratliff-Rush. (For, given any positive integer $n$, let $x \in I^{n+1}: I^{n}$; then $x a^{n} \in x I^{n} \subseteq$ $I^{n+1}=a^{n} I$, and since $a$ is a nonzerodivisor, we see that $x \in I$.) Since the set of stable ideals is closed under product (for, if $I^{2}=a I$ and $J^{2}=b J$, then $(I J)^{2}=(a b)(I J)$ ), all powers of $I$ are also Ratliff-Rush ideals. On the other hand, if the regular ideal $I$ in the Noetherian $\operatorname{ring} R$ has a reduction $J$ for which $J I$ is Ratliff-Rush, then $I$ has reduction number at most one with respect to $J$. (For, if $J I^{n}=I^{n+1}$, then $(J I)^{n+k}=\left(I^{2}\right)^{n+k}$ for all $k \geq 0$, so $I^{2} \subseteq \widetilde{J I}=J I \subseteq I^{2}$.) In particular, if $I$ has a principal reduction $a R$ and $a I$ is Ratliff-Rush, then $I$ is stable and all powers of $I$ are Ratliff-Rush.

(1.2) For a proper regular ideal $I$ in the Noetherian ring $R$, we denote by $\mathrm{G}(I)$ the graded ring (or form ring) $R / I \oplus I / I^{2} \oplus I^{2} / I^{3} \oplus \ldots$ All powers of $I$ are Ratliff-Rush ideals iff its positively graded ideal $\mathrm{G}(I)^{+}=$ $I / I^{2} \oplus I^{2} / I^{3} \oplus \ldots$ contains a nonzerodivisor. For, if $\mathrm{G}(I)^{+}$contains a nonzerodivisor, then $\mathrm{G}(I)^{+}$is not contained in any associated prime of 0 in $G(I)$, and hence neither is $I / I^{2}$. Hence for some positive integer $s$, there exists a nonzerodivisor $a^{*}$ in $I^{s} / I^{s+1}$. If $a$ is a preimage of $a^{*}$ in $I^{s}$, then $I^{n+k s}: a^{k}=I^{n}$ for all positive integers $n, k$, so $I^{n+k s}: I^{k s}=I^{n}$ for all $n, k$, 
and hence by [RR, (2.3.1)] all powers of $I$ are Ratliff-Rush. Conversely, if $\mathrm{G}(I)^{+}$consists of zerodivisors, then $G(I)^{+}$is contained in some associated prime of 0 . Hence for some nonnegative integer $s$ there exists a homogeneous element $a^{*}$ in $I^{s} / I^{s+1}$ such that $a^{*} \mathrm{G}(I)^{+}=0$. If $a$ is a preimage of $a^{*}$ in $I^{s}$ and $J=I^{s+1}$, then $a J \subseteq J^{2}$, so $a \in \widetilde{J}-J$. (In [Sh, Theorem 4], Shah provides a proof for this fact in the case that $(R, M)$ is quasi-unmixed local, $R / M$ is infinite, and $I$ is $M$-primary.) In particular, these conditions hold for the stable ideals of (1.1). Also, if $I$ is generated by a regular sequence (or more generally by a quasiregular sequence in the sense of Matsumura [M, pages 124-125]), then since $\mathrm{G}(I)$ is (isomorphic to) a polynomial ring over $R / I$ with indeterminates the images of the generators, $I$ and its powers are Ratliff-Rush ideals; indeed, $I^{n+k}: I^{k}=I^{n}$ for all positive integers $n, k$.

(1.3) If $(R, M)$ is a Cohen-Macaulay local ring of positive dimension and $I$ is an $M$-primary ideal with reduction number at most one, then every power of $I$ is a Ratliff-Rush ideal. For, by [V, Lemma 1 and Theorem 1], the graded ring $\mathrm{G}(I)$ is Cohen-Macaulay; in particular, $\mathrm{G}(I)^{+}$contains a nonzerodivisor, so $I$ and all its powers are Ratliff-Rush ideals.

(1.4) Let $(R, M)$ be a local ring and $I$ a regular $M$-primary ideal. Since $\widetilde{I}$ is the unique largest ideal in $R$ with the property that $(\widetilde{I})^{n}=I^{n}$ for sufficiently large $n$, in this setting $\widetilde{I}$ can be characterized as the unique largest ideal containing $I$ and having the same Hilbert polynomial $P_{I}(n)$ (= length $\left(R / I^{n}\right)$ for sufficiently large $n$ ) as $I$. If $R$ is quasi-unmixed of dimension $d$ and $R / M$ is infinite, it is shown in [Sh] that there exist unique largest ideals $I_{k}, k=1, \ldots, d$, such that $I \subseteq I_{d} \subseteq \ldots \subseteq I_{1} \subseteq I^{\prime}$ (where $I^{\prime}$ denotes the integral closure of $I$ ) and the top $k+1$ coefficients of the Hilbert polynomials of $I_{k}$ agree with those of $I$. It follows from their maximality that each of these coefficient ideals of $I$ is a Ratliff-Rush ideal.

(1.5) Let $R, S$ be Noetherian rings and $f: R \rightarrow S$ define $S$ as an $R$ algebra. Suppose $I$ is a regular ideal in $R$ such that $I S$ is a Ratliff-Rush ideal in $S$ and $I=f^{-1}(I S)$. Then $I$ is Ratliff-Rush in $R$. (For, if $a \in I^{n+1}:_{R} I^{n}$, then $f(a) \in(I S)^{n+1}:_{S} I^{n}=(I S)^{n+1}:_{S}(I S)^{n}$, so $f(a) \in I S$ because $I S$ is Ratliff-Rush, so $a \in I$.) In particular, if $f: R \rightarrow S$ is surjective, then the preimage in $R$ of a Ratliff-Rush ideal in $S$, if regular, is itself RatliffRush. The surjectivity hypothesis is necessary here: Example 1.6 below shows that, in general, a nonzero ideal $I$ in a Noetherian domain $R$ may be the contraction of a Ratliff-Rush ideal in a finitely generated birational integral extension of $R$, and yet $I$ is not a Ratliff-Rush ideal. 
Example 1.6. Let $R=k\left[\left[t^{4}, t^{5}\right]\right], S=R\left[t^{6}\right]$, and $J=t^{11} S$. Then $J$ is a Ratliff-Rush ideal in $S$, but $I=J \cap R=\left(t^{15}, t^{16}, t^{17}\right) R$ is not Ratliff-Rush since $t^{18} \in R-I$ and $I^{2}=\left(I+t^{18} R\right)^{2}$.

(1.7) Let $R, S$, and $f$ be as in (1.5). Suppose that $S$ is flat over $R$. Then for any regular ideal $I$ in $R, \widetilde{I S}=\widetilde{I} S$, because $(I S)^{n+1}:_{S}(I S)^{n}=\left(I^{n+1}:_{R}\right.$ $\left.I^{n}\right) S$ [M, Theorem 7.4, page 48]. In particular, if $I$ is Ratliff-Rush, then so is $I S$. It follows that $I$ is Ratliff-Rush iff $I R_{P}$ is Ratliff-Rush for every prime ideal or every maximal ideal $P$ of $R$ [LM, Proposition 3.13, page 70] [AM, Proposition 3.8, page 40]. In fact, $I$ is Ratliff-Rush iff $I R_{P}$ is RatliffRush for every associated prime $P$ of $I$ [M, Exercise 6.4, page 42]. Now, suppose $S$ is faithfully flat over $R$ (so $f$ may be regarded as an inclusion); then $\widetilde{I}=\widetilde{I S} \cap R$ for any regular ideal $I$ in $R$, and $I S$ is a Ratliff-Rush ideal in $S$ iff $I$ is Ratliff-Rush in $R$. This follows because faithful flatness assures that every ideal of $R$ is the contraction of its extension to $S$ [M, Theorem 7.5, page 49].

(1.8) Let $I$ be a regular ideal in a Noetherian ring $R$, and let $a$ be an element of $I^{s}-I^{s+1}$ such that the image $a^{*}$ of $a$ in $I^{s} / I^{s+1} \subseteq \mathrm{G}(I)$ is a nonzerodivisor. (Thus, all powers of $I$ are Ratliff-Rush ideals.) Then $J=I^{s+1}+a R$ is also a Ratliff-Rush ideal. To see this, we note first that the hypothesis on $a^{*}$ means that $I^{k+s}: a=I^{k}$ for each positive integer $k$. Now since it is clear that, for every nonnegative integer $n, J \subseteq J^{n+1}: J^{n} \subseteq J^{n+1}: a^{n}$, the result will follow when we show that $J^{n+1}: a^{n} \subseteq J$. We proceed by induction on $n$, the case $n=0$ being clear. So assume $n>0$ and $J^{n}: a^{n-1} \subseteq J$, and let $x \in J^{n+1}: a^{n}$. Write $a^{n} x=\sum_{j=0}^{n+1} a^{j} b_{j}$ where $b_{j} \in\left(I^{s+1}\right)^{n+1-j}$. Then since $a\left(a^{n-1} x-\sum_{j=1}^{n+1} a^{j-1} b_{j}\right)=b_{0} \in\left(I^{s+1}\right)^{n+1}$, the hypothesis on $a^{*}$ shows that $a^{n-1} x-\sum_{j=1}^{n+1} a^{j-1} b_{j} \in I^{(s+1)(n+1)-s} \subseteq\left(I^{s+1}\right)^{n}$, so that (writing $k=j-1$ ) we get $a^{n-1} x \in \sum_{k=0}^{n} a^{k}\left(I^{s+1}\right)^{(n+1)-(k+1)}+\left(I^{s+1}\right)^{n}=$ $\sum_{k=0}^{n} a^{k}\left(I^{s+1}\right)^{n-k}=J^{n}$, and we see that $x \in J^{n}: a^{n-1} \subseteq J$.

(1.9) If $I, J$ are comaximal Ratliff-Rush ideals, then their product is again Ratliff-Rush. For, since the powers of $I$ are comaximal with the 
powers of $J$, so that products are intersections, we have:

$$
\begin{aligned}
(I J)^{n+1}:(I J)^{n} & =\left(I^{n+1} \cap J^{n+1}\right): I^{n} J^{n} \\
& =\left(\left(I^{n+1}: I^{n}\right): J^{n}\right) \cap\left(\left(J^{n+1}: J^{n}\right): I^{n}\right) \\
& =\left(I: J^{n}\right) \cap\left(J: I^{n}\right) \\
& =\left(I:\left(I+J^{n}\right)\right) \cap\left(J:\left(J+I^{n}\right)\right) \\
& =(I: R) \cap(J: R) \\
& =I \cap J \\
& =I J .
\end{aligned}
$$

(1.10) It should be noted that, although the process of passing from $I$ to $\widetilde{I}$ has some of the properties of a $*$-operation on ideals as described in [G, $\S 32]$ (or a '-operation in [ZS, page 355]), it does not have all these properties. In particular, let $I$ be an ideal in a Noetherian domain $R$, and let $a \in R$. Then it need not be the case that $\widetilde{a I}=a \widetilde{I}$. Indeed, even if $I$ and all its powers are Ratliff-Rush and $a R$ is a minimal reduction of $I$, then by (1.1) if the reduction number of $I$ is greater than one (i.e., $I$ is not stable), $a I$ will not be Ratliff-Rush. Therefore the family of Ratliff-Rush ideals of an integral domain is not closed, in general, under isomorphism. Nor is it closed under intersection; in particular, $I \subseteq J$ does not imply $\widetilde{I} \subseteq \widetilde{J}$. The following example exhibits both these phenomena. (We use the symbol $<$ between sets to denote proper inclusion.)

Example 1.11. Let $R=k\left[\left[t^{3}, t^{4}\right]\right]$, where $t$ is an indeterminate over the field $k$, and let $M=\left(t^{3}, t^{4}\right) R$, the maximal ideal of $R$. Then since $R \cong$ $k[[x, y]] /\left(y^{3}-x^{4}\right)$ and the graded ring of $(x, y) k[[x, y]]$ in $k[[x, y]]$ is (isomorphic to) $k[x, y]$, it follows from [M, Exercise 14.3, page 115] or $[\mathrm{Ku}$, Lemma 5.3, page 150] that $\mathrm{G}(M) \cong k[x, y] /\left(y^{3}\right) k[x, y]$. Thus $\mathrm{G}(M)$ is Cohen-Macaulay, so $\mathrm{G}(M)^{+}$contains a nonzerodivisor. It follows that all the powers of $M$ are Ratliff-Rush ideals. We have $t^{8} \in \widetilde{t^{3} M}$, since $\left(t^{3} M\right)^{2}=M^{4}$, but $t^{8} \notin t^{3} M$, so $t^{3} M<\widetilde{t^{3} M}$. Moreover, the ideal $t^{8} M=$ $\left(t^{11}, t^{12}\right) R=t^{3} R \cap t^{8} R$ is an intersection of two principal (and hence RatliffRush) ideals of $R$, but $t^{13} \in\left(t^{11}, t^{12}, t^{13}\right) R-t^{8} R \subseteq \widetilde{t^{8} M}-t^{8} M$.

(1.12) In certain cases, however, we can see that the product of a regular principal ideal $a R$ and a Ratliff-Rush ideal $I$ is again Ratliff-Rush:

(1) Since stable ideals are closed under product, the product of two stable ideals (one of which can be principal) is again Ratliff-Rush by (1.1). 
(2) If $a R$ is a "root-closed" ideal (i.e., $b^{n} \in a^{n} R$ implies $b \in a R$ ), then for any Ratliff-Rush ideal $I$ of $R, a I$ is again Ratliff-Rush. (For, if $b \in \widetilde{a I}$, say $(b R+a I)^{n}=(a I)^{n}$, then $b^{n} \in(a I)^{n} \subseteq a^{n} R$, so $b=a r$, say; and $(r R+I)^{n}=I^{n}$, so since $I$ is Ratliff-Rush, $r \in I$ and $b \in a I$.) We remark that if $a$ is a product of prime elements in a Noetherian domain $R$, then $a R$ is a root-closed ideal. Also nonzero principal ideals in an integrally closed domain are integrally closed and hence root-closed. Thus, in an integrally closed Noetherian domain, the product of a nonzero principal ideal and a Ratliff-Rush ideal is again Ratliff-Rush.

(3) On the other hand, for a nonzero ideal $I$ and a nonzerodivisor $a$ in $R$, if $a I$ is Ratliff-Rush, then $I$ is Ratliff-Rush. For, if $I$ is not RatliffRush, say $I<I^{n+1}: I^{n}$, then $a I<a\left(I^{n+1}: I^{n}\right) \subseteq(a I)^{n+1}:(a I)^{n}$, so $a I$ is also not Ratliff-Rush.

(1.13) It would be interesting to have conditions on a Noetherian domain $R$ in order that the powers of any Ratliff-Rush ideal in $R$ again be RatliffRush. By (1.2), this is equivalent to the condition that for each proper Ratliff-Rush ideal $I$ in $R$, the positively graded ideal $\mathrm{G}(I)^{+}$of the graded ring $\mathrm{G}(I)$ contains a nonzerodivisor. It would also be interesting to know when the family of Ratliff-Rush ideals in $R$ is closed under product, or when every Ratliff-Rush ideal in $R$ is stable. (By (1.1), if every Ratliff-Rush ideal in $R$ is stable, then the Ratliff-Rush ideals in $R$ are closed under product.) Lipman shows in [L, Theorem 2.2] that a one-dimensional local domain $R$ is an Arf ring iff every integrally closed ideal in $R$ is stable, or equivalently iff for every local ring $S$ infinitely near to $R$ the embedding dimension of $S$ is equal to the multiplicity of $S$. It would be interesting to know for which Arf rings $R$ it is the case that every Ratliff-Rush ideal in $R$ is stable.

(1.14) Let $(R, M)$ be a one-dimensional Cohen-Macaulay local ring and let $I$ be an $M$-primary ideal in $R$. Lipman in [L, Corollary 1.4] shows that all sufficiently high powers of $I$ are stable, and that $I^{n}$ is stable iff length $\left(I^{s} / I^{s+1}\right)$ is the multiplicity of $I$ for all $s \geq n$ [L, Corollary 1.6]. In particular, the maximal ideal $M$ is stable iff the minimal number of generators of the powers of $M$ is constant.

(1.15) Let $I$ be a regular ideal in a local ring $R$. Eakin and Sathaye [ES, Corollary 1, page 446] show that the following statements are equivalent: (i) Some power of $I$ is stable. (ii) There is a bound on the number of generators of powers of $I$. (iii) For some positive integer $n, I^{n}$ can be 
generated by $n$ elements. Sally and Vasconselos [SV2, proof of Theorem 3.4] show that if both $I$ and $I^{2}$ are two-generated, then $I$ is stable. Example 1.16 shows, however, that it can happen, that all powers of $I$ are minimally generated by three elements, and yet $I$ is not stable or even Ratliff-Rush.

Example 1.16. Let $R=k\left[\left[t^{3}, t^{10}, t^{11}\right]\right]$ and $I=\left(t^{9}, t^{10}, t^{14}\right) R$. Then $\widetilde{I}=$ $\left(t^{9}, t^{10}, t^{11}\right) R$, so $I$ is not Ratliff-Rush. But all powers of $I$ are minimally generated by three elements.

(1.17) Let $(R, M)$ be a one-dimensional local domain. We observe that the fact that $M$ is stable does not imply that $R$ is an Arf ring or that powers of integrally closed ideals are necessarily Ratliff-Rush.

(i) In $R=k\left[\left[t^{3}, t^{7}, t^{11}\right]\right]$, we have $M^{2}=t^{3} M$, and the ideal $I=\left(t^{6}, t^{7}, t^{11}\right) R$ is integrally closed, but it is not stable, since $t^{14} \in I^{2}-t^{6} I$.

(ii) In $R=k\left[\left[t^{4}, t^{11}, t^{17}, t^{18}\right]\right]$, we have $M^{2}=t^{4} M$, and the ideal $I=$ $\left(t^{11}, t^{12}, t^{17}, t^{18}\right) R$ is integrally closed, but $I^{2}$ is not integrally closed or even Ratliff-Rush, since $t^{25} I^{2} \subset I^{4}$, so $t^{25} \in \widetilde{I^{2}}-I^{2}$.

(1.18) The Briançon-Skoda theorem(s) [LS] state(s) that, under some hypotheses of regularity, the integral closure of a certain power of an ideal is contained in the ideal. Since the Ratliff-Rush ideal associated to an ideal is contained in the integral closure, it follows that these hypotheses also imply that the Ratliff-Rush ideal associated to that power is also contained in the ideal. It would be interesting to have information more generally on the smallest positive integer $n$ such that $\widetilde{I^{n}} \subseteq I$. If $(R, M)$ is a one-dimensional local domain having multiplicity $e>1$, then by [SV2, Proposition 2.2 and Theorem 2.5], for each nonzero ideal $I$ of $R, I^{e-1}$ is stable and hence Ratliff-Rush, so $\widetilde{I^{e-1}} \subseteq I$. But it is not true in general for an ideal $I$ of a one-dimensional local domain $R$ that $\widetilde{I^{2}} \subseteq I$ : For example, suppose $R=k\left[\left[t^{5}, t^{6}\right]\right], M=\left(t^{5}, t^{6}\right) R$ (the maximal ideal of $R$ ), and $I=t^{5} M=\left(t^{10}, t^{11}\right) R$. Then $t^{24} \in M^{4}=\widetilde{I}^{2}$, but $t^{24} \notin I$.

(1.19) To see why it is natural to require that Ratliff-Rush ideals be regular, we observe that the generator of a principal ideal in a nonzero Noetherian ring satisfying the Ratliff-Rush condition is a nonzerodivisor: It suffices to show this in localizations at maximal ideals containing the principal ideal. So suppose $a$ is a nonunit in a (Noetherian) local ring $R$ such that $a R=\bigcup\left\{a^{n+1} R:_{R} a^{n} R \mid n \geq 1\right\}$ (so $a \neq 0$, because $R \neq 0$ ), and that $a b=0$. Then $b \in a^{2} R: a R=a R$, say $b=a b_{1}$. Since $a^{2} b_{1}=0$, we see that $b_{1} \in a^{3} R: a^{2} R=a R$, say $b_{1}=a b_{2}$. Continuing in this way, we see that $b \in \bigcap\left\{a^{n} R \mid n \geq 1\right\}=0$; so $a$ is a nonzerodivisor. 
2. The associated Ratliff-Rush ideal as a contraction. In this section, $R$ will always denote a Noetherian domain. We begin with some results closely related to Corollary 3.10 of [MRR] and Theorem 2.4 of [MR].

Fact 2.1. Let I be a nonzero proper ideal in a Noetherian domain $R$. The associated Ratliff-Rush ideal $\tilde{I}$ of $I$ is the contraction to $R$ of the extension of $I$ to its "blowup" $\mathcal{B}(I)=\left\{R[I / a]_{P} \mid a \in I-0, P \in \operatorname{Spec}(R[I / a])\right\}$ (i.e., $\widetilde{I}=\bigcap\{I S \cap R \mid S \in \mathcal{B}(I)\}$, or equivalently $\widetilde{I}=\bigcap\{I R[I / a] \cap R \mid a \in I-0\})$.

Recall. $\mathcal{B}(I)$ is the set of elements minimal with respect to domination among the local birational extensions $S$ of $R$ for which $I S$ is principal.

Proof. Let $x \in \widetilde{I}$, i.e., $x I^{n} \subseteq I^{n+1}$ for some $n$. For each $S$ in $\mathcal{B}(I), I S$ is principal, say $I S=a S$ for some $a$ in $I-0$. Thus, $x a^{n} S \subseteq a^{n+1} S$, and so $x \in a S=I S$. Since $S$ was any element of $\mathcal{B}(I), x$ is in the contraction of the extension of $I$ to its blowup.

Conversely, suppose $x$ is in the contraction of $I$ from its blowup. Take $a$ in $I-0$; since $x \in I R[I / a]_{P}=a R[I / a]_{P}$ for each $P$ in $\operatorname{Spec}(R[I / a])$, $x \in a R[I / a]$ [LM, Proposition 3.13, page 70] [AM, Proposition 3.8, page 40], i.e., $x=a f\left(b_{1} / a, \ldots, b_{n} / a, 1\right)$ where $b_{1}, \ldots, b_{n}$ is a generating set for $I$ and $f$ is a homogeneous polynomial in $n+1$ variables over $R$, of degree $n(a)$, say. Multiplying both sides by $a^{n(a)}$ shows $x a^{n(a)}=a f\left(b_{1}, \ldots, b_{n}, a\right) \in I^{n(a)+1}$. Thus if $m=n\left(b_{1}\right)+\ldots+n\left(b_{n}\right)$, we have $x I^{m} \subseteq I^{m+1}$, i.e., $x \in \widetilde{I}$.

(2.2) For a more careful analysis of the Ratliff-Rush ideal $\widetilde{I}$ associated to $I$, it is desirable to sharpen Fact 2.1 to get $\widetilde{I}$ to be contracted from one affine piece of the blowup of $I$. In $\mathrm{G}(I)$, write a primary decomposition of the zero ideal: $0=\mathbf{q}_{1} \cap \ldots \cap \mathbf{q}_{s} \cap Q_{1} \cap \ldots \cap Q_{t}$, where the radicals of the primary ideals $Q_{i}$ contain $\mathrm{G}(I)^{+}$and the radicals of the primary ideals $\mathbf{q}_{i}$ do not contain $\mathrm{G}(I)^{+}$. (Note that $\mathrm{G}(I)^{+}$is not nilpotent in $\mathrm{G}(I)$, since the powers of $I$ properly descend. Hence there is at least one $\mathbf{q}_{i}$.) Then $\widetilde{I}$ is the preimage in $R$ of $\mathbf{q}_{1} \cap \ldots \cap \mathbf{q}_{s} \cap R / I$, where $R / I$ is regarded as the 0 -th homogeneous piece of $\mathrm{G}(I)$. For, it is clear that $\widetilde{I}$ is the preimage in $R$ of the annihilator of $\left(\mathrm{G}(I)^{+}\right)^{k}$ for sufficiently large $k$; and for large $k$ we have 
$\left(\mathrm{G}(I)^{+}\right)^{k} \subseteq Q_{1} \cap \ldots \cap Q_{t}$, so taking colons in $\mathrm{G}(I)$, we have

$$
\begin{aligned}
0:\left(G(I)^{+}\right)^{k}= & \left(\mathbf{q}_{1} \cap \ldots \cap \mathbf{q}_{s} \cap Q_{1} \cap \ldots \cap Q_{t}\right):\left(G(I)^{+}\right)^{k} \\
= & \left(\mathbf{q}_{1}:\left(G(I)^{+}\right)^{k}\right) \cap \ldots \cap\left(\mathbf{q}_{s}:\left(G(I)^{+}\right)^{k}\right) \\
& \cap\left(Q_{1}:\left(G(I)^{+}\right)^{k}\right) \cap \ldots \cap\left(Q_{t}:\left(G(I)^{+}\right)^{k}\right) \\
= & \mathbf{q}_{1} \cap \ldots \cap \mathbf{q}_{s} \cap G(I) \\
= & \mathbf{q}_{1} \cap \ldots \cap \mathbf{q}_{s} .
\end{aligned}
$$

Since an ideal contained in a finite union of primes is contained in one of them, for some positive integer $n$ there exists an element $a$ of $I^{n}-I^{n+1}$ for which the image $a^{*}$ in $I^{n} / I^{n+1} \subseteq \mathrm{G}(I)$ is not in the radical of any $\mathbf{q}_{i}$. Then as above $0:\left(a^{*}\right)^{k}=\mathbf{q}_{1} \cap \ldots \cap \mathbf{q}_{s}$ for sufficiently large $k$. By the description of $\widetilde{I}$ in terms of the $\mathbf{q}_{i}$ 's, it follows that $I^{n k+1}: a^{k}=\widetilde{I}$. Therefore $\widetilde{I}=I R\left[I^{n} / a\right] \cap R$, the contraction of the extension of $I$ to one affine piece $R\left[I^{n} / a\right]$ of the blowup $\mathcal{B}\left(I^{n}\right)=\mathcal{B}(I)$. Also, if we let $P$ vary over the associated primes of $I R\left[I^{n} / a\right]$, we see that $\widetilde{I}$ is the contraction to $R$ of the extension of $I$ to a finite number of the local rings in $\mathcal{B}(I)$. (We remark that the above condition on $a$ is equivalent to the statement that $a$ is superficial of order $n$ for $I$, in the sense of [ZS, page 285]. Our discussion of an ideal in a Noetherian domain is similar to theirs of an open ideal in a semilocal ring. Also, it is interesting to compare the realization of $\widetilde{I}$ as the colon ideal $I^{n k+1}: a^{k}$ with the result of Shah in [S, Theorem 3]: If $(R, M)$ is a quasi-unmixed local ring of dimension $d$ and $I$ is an $M$-primary ideal with associated coefficient ideals $I_{k}, k=1, \ldots, d$, as in (1.4), then for a certain minimal reduction $\left(x_{1}, \ldots, x_{d}\right) R$ of a certain power $I^{n}$ of $I$, $I_{k}=I^{n}:\left(x_{1}, \ldots, x_{k}\right) R$ for each $k=1, \ldots, d$. $)$

(2.3) If $I$ is a nonzero proper ideal of a one-dimensional local domain $(R, M)$, then the blowup $\mathcal{B}(I)$ has an especially nice form. The ideal $I$ may not have a principal reduction; but as noted in (1.14), some power $I^{n}$ of $I$ is stable, so $I^{n}$ does have a principal reduction, say $a R$. The blowup $\mathcal{B}\left(I^{n}\right)=\mathcal{B}(I)$ regarded as a model [ZS, page 116] is affine over $R$ with $R\left[I^{n} / a\right]$ as ring of global sections, i.e., all the rings in this blowup are localizations of the single affine piece $R\left[I^{n} / a\right]$. (This follows because if $S$ is a local ring between $R$ and its field of fractions for which $I^{n} S$ is principal, then the fact that $a R$ is a principal reduction of $I^{n}$ implies $I^{n} S=a S$, and hence that $R\left[I^{n} / a\right] \subseteq S$.) Thus, $I$ or any power $I^{k}$ of $I$ is Ratliff-Rush iff it is contracted from $R\left[I^{n} / a\right]$. In what follows, when we speak of the blowup of a nonzero proper ideal of a one-dimensional local domain, we will mean 
this ring of global sections. We remark that if a suitably large power $I^{n}$ of $I$ is taken so that $I^{n}$ is stable, and if $a$ in $I^{n}$ is such that $a I^{n}=I^{2 n}$, then the blowup ring $R\left[I^{n} / a\right]$ is the fractional $R$-ideal $I^{n} / a$, and the minimal number of generators of $I^{n}$ as an ideal in $R$ is the same as the minimal number of generators of $R\left[I^{n} / a\right]$ as an $R$-module. Since the blowup of $I$ is a finitely generated $R$-module, it is a subring of the integral closure of $R$. We also note that since the extension of $I$ to its blowup has a principal power, and the blowup is semilocal, the extension of $I$ must be invertible and therefore principal [Ka, Theorem 60, page 37]; it may not be possible, however, to choose a generator from the elements of $I$ if the residue field $R / M$ of $R$ is finite and the integral closure $R^{\prime}$ of $R$ has more maximal ideals than the cardinality of $R / M$. Indeed, $I$ has a principal reduction iff $I R^{\prime}$ is generated by an element of $I$. (We remark that, in one of the power series rings $R$ described in the introduction, if an ideal $I$ generated by monomials has order $n$, then the blowup of $I$ is formed by adjoining to $R$ the elements $t^{m-n}$ as $t^{m}$ varies over the other generators of $I$. In particular, if $I$ has order $n$ and $t^{n+1} \in I$, then the blowup of $I$ is the integral closure $k[[t]]$ of $R$, so $\widetilde{I}$ is the integral closure of $I$; cf. Corollary 2.7 below.)

The observations in the above paragraph imply the following:

Proposition 2.4. Let $(R, M)$ be a one-dimensional local domain and let $R^{\prime}$ denote the integral closure of $R$. If $I$ and $J$ are stable ideals in $R$ with principal reductions $a R$ and $b R$, respectively, and if $I$ and $J$ have the same blowup, then $I / a=R[I / a]=R[J / b]=J / b$, so $b I=a J$. Conversely, if $S$ is a subring of $R^{\prime}$ containing $R$ and finitely generated as an $R$-module, say by $1, b_{1} / a, \ldots, b_{n} / a$ where $a, b_{i} \in R$, then $S$ is the blowup of the stable ideal $\left(a, b_{1}, \ldots, b_{n}\right) R$. It follows that the subrings of $R^{\prime}$ containing $R$ that are finitely generated $R$-modules are in one-to-one correspondence with the isomorphism classes of stable ideals of $R$. In particular, there are only finitely many isomorphism classes of stable ideals of $R$ iff there are only finitely many subrings of $R^{\prime}$ containing $R$ that are finitely generated $R$ modules.

Example 2.5. Suppose $k$ is an infinite field and $R=k\left[\left[t^{4}, t^{5}\right]\right]$. For $a \in k$, let $S_{a}=R\left[t^{2}+a t^{3}\right]$. If $a, b$ are distinct elements of $k$, then $S_{a} \cap S_{b}=$ $R$, while $S_{a}\left[S_{b}\right]=k\left[\left[t^{2}, t^{3}\right]\right]$. Therefore $\left\{S_{a}\right\}_{a \in k}$ is an infinite family of distinct finitely generated $R$-subalgebras of $k[[t]]$. Hence there are infinitely many isomorphism classes of stable ideals in $R$. On the other hand, if $S=k\left[\left[t^{3}, t^{4}\right]\right]$, the only rings properly between $S$ and $k[[t]]$ are the rings $S\left[t^{5}\right]$ 
and $S\left[t^{2}\right]$. Hence there are (including nonzero principal ideals) precisely four distinct isomorphism classes of stable ideals in $S$. The ring $k\left[\left[t^{3}, t^{4}\right]\right]$ is considered in more detail in Example 2.14 below.

Proposition 2.6. Let $I$ be a nonzero proper ideal of a one-dimensional local domain $R$, and let $S$ be the blowup of $I$. If $T$ is an $R$-subalgebra of $R^{\prime}$ such that $I^{n} T \cap R \subseteq \widetilde{I^{n}}$ for infinitely many positive integers $n$, then $T \subseteq S$. Therefore, $S$ is the unique maximal subring of the integral closure $R^{\prime}$ of $R$ for which $\widetilde{I^{n}}=I^{n} S \cap R$ for each positive integer $n$,

Proof. It follows from Fact 2.1 that $\widetilde{I^{n}}=I^{n} S \cap R$ for each positive integer $n$. Let $T$ be an $R$-subalgebra of $R^{\prime}$ for which $I^{n} T \cap R \subseteq \widetilde{I^{n}}$ for infinitely many $n$; to see that $T \subseteq S$, it suffices to show this when $T$ is finitely generated over $R$. Let $a$ be a generator of $I S$. Then $a$ is in the Jacobson radical of $S$, so for sufficiently large $n, a^{n}$ is in the conductors of both $T$ and $S$ into $R$. Thus, for some $n, a^{n} T \subseteq I^{n} T \cap R \subseteq \widetilde{I^{n}}=I^{n} S \cap R=a^{n} S \cap R=a^{n} S$. Cancelling $a^{n}$ yields the result.

In the proof of the following corollary, we use the fact that in a onedimensional Noetherian domain $R$, the integral closure $I^{\prime}$ of an ideal $I$ is the contraction to $R$ of the extension of $I$ to the integral closure $R^{\prime}$ of $R$. This follows from the fact that every ideal in the Dedekind domain $R^{\prime}$ is integrally closed.

Corollary 2.7. Let $(R, M)$ be a one-dimensional local domain with integral closure $R^{\prime}$, and let $I$ be a nonzero proper ideal of $R$. Then the following conditions are equivalent:

(i) $\widetilde{I^{n}}=\left(I^{n}\right)^{\prime}$, the integral closure of $I^{n}$, for all positive integers $n$.

(ii) $\widetilde{I^{n}}=\left(I^{n}\right)^{\prime}$ for infinitely many $n$.

(iii) The blowup of $I$ is $R^{\prime}$.

In particular, $R^{\prime}$ is a finitely generated $R$-module iff there exists a nonzero proper ideal I such that $\widetilde{I^{n}}=\left(I^{n}\right)^{\prime}$ for infinitely many positive integers $n$, or equivalently, such that all sufficiently high powers of I are integrally closed.

Proof. (i) $\Rightarrow$ (ii): Clear. (ii) $\Rightarrow$ (iii): Apply Proposition 2.6 and the above remark. (iii) $\Rightarrow$ (i): Apply the above remark.

We remark that for $R$ as in (2.7), the fact that an ideal $I$ in $R$ is integrally closed, or even integrally closed and stable, does not imply that the blowup of $I$ is $R^{\prime}$. For example, if $R=k\left[\left[t^{3}, t^{5}, t^{7}\right]\right]$, then the maximal ideal $M=$ $\left(t^{3}, t^{5}, t^{7}\right) R$ is integrally closed and stable, but the blowup of $M$ is $k\left[\left[t^{2}, t^{3}\right]\right]$. 
Ratliff and Rush [RR, (2.5)] note that a domain in which every ideal is either principal or integrally closed is one in which every nonzero ideal is what we are calling a Ratliff-Rush ideal. The following corollary of Proposition 2.4 describes the one-dimensional local domains in which every RatliffRush ideal is either principal or integrally closed. But in Example 2.10 (ii) below we show that this property does not imply that every ideal is either principal or integrally closed.

Theorem 2.8. Let $(R, M)$ be a one-dimensional local domain. Every RatliffRush ideal of $R$ is either principal or integrally closed iff there are no rings properly between $R$ and its integral closure $R^{\prime}$.

Proof. If there are no rings properly between $R$ and $R^{\prime}$, then since the blowup of a nonprincipal ideal properly contains $R$, the blowup of a nonprincipal Ratliff-Rush ideal must be $R^{\prime}$, so by Corollary 2.7 the ideal is integrally closed. Conversely, if there are nonzero elements $a, b$ of $R$ for which $R<R[a / b]<R^{\prime}$, then all but finitely many powers of the ideal $(a, b) R$ have associated Ratliff-Rush ideals that are neither principal (for, no principal ideal can be contracted from a properly larger extension within the field of fractions) nor integrally closed (by Corollary 2.7).

(2.9) Suppose that $(R, M)$ is a one-dimensional local domain and there are no rings properly between $R$ and its integral closure $R^{\prime}$. Then $R^{\prime}$ has at most two maximal ideals; for, if $R^{\prime}$ had three distinct maximal ideals and $x$ were taken to be in two of these maximal ideals but not the third, then two of the maximal ideals in $R^{\prime}$ would have the same intersection $M+x R[x]$ with $R[x]$, but $R[x]$ would have at least two maximal ideals, so $R[x]$ would be a ring properly between $R$ and $R^{\prime}$. Suppose first that $R^{\prime}$ has two maximal ideals. Then $R^{\prime} / M R^{\prime}$ has dimension 2 as a vector space over $R / M$ : For, if $M R^{\prime}$ has primary decomposition $M R^{\prime}=Q_{1} \cap Q_{2}$, then $R^{\prime} / M R^{\prime} \cong R^{\prime} / Q_{1} \oplus R^{\prime} / Q_{2}$; if either summand, say $R^{\prime} / Q_{1}$, properly contained its image of $R / M$, then the preimage $R+Q_{1}$ in $R^{\prime}$ of $R / M$ in $R / Q_{1}$ would be a ring properly between $R$ and $R^{\prime}$. Therefore $R$ has multiplicity two [ZS, page 300], so by Corollary 3.6 below, it follows that every nonzero ideal in $R$ is a Ratliff-Rush ideal. Now suppose that $R^{\prime}$ is local (and hence a discrete rank-one valuation domain - which we abbreviate, as usual, by DVR - with principal maximal ideal $M^{\prime}$ ) and the residue field $R / M$ of $R$ is algebraically closed. Then the residue field of $R^{\prime}$ is algebraic over and hence equal to $R / M$. Thus, either (1) $M R^{\prime}=M^{\prime}$, and hence $R=R^{\prime}$ by Nakayama's Lemma [M, Corollary, page 8]; or (2) $M R^{\prime}=\left(M^{\prime}\right)^{2}$, for, if 
$M R^{\prime}<\left(M^{\prime}\right)^{2}$, then $R+\left(M^{\prime}\right)^{2}$ would be a ring properly between $R$ and $R^{\prime}$. In the latter case $R^{\prime} / M R^{\prime}=R^{\prime} /\left(M^{\prime}\right)^{2}$ again has dimension 2 as a vector space over $R / M$ (with basis the images of 1 and a generator of $M^{\prime}$ ), $R$ has multiplicity two [ZS, page 300], and every nonzero ideal in $R$ is RatliffRush. On the other hand, as we illustrate in Example 2.10 (ii), if the residue field of $R$ is not algebraically closed, then the condition that every RatliffRush ideal in $R$ be either principal or integrally closed does not restrict the multiplicity of $R$ and $R$ may have nonzero ideals that are not Ratliff-Rush.

Examples 2.10. One-dimensional local domains $R$ for which there are no rings properly between $R$ and its integral closure:

(i) Let $k$ be any field and $t$ be an indeterminate. Then the local rings of the cusp $k\left[t^{2}, t^{3}\right]_{\left(t^{2}, t^{3}\right)}$ and of the node $k\left[t(t-1), t^{2}(t-1)\right]_{\left(t(t-1), t^{2}(t-1)\right)}$ have the desired properties. In these two examples, the ring has multiplicity two, and every nonzero ideal in $R$ is Ratliff-Rush.

(ii) For any integer $n>1$ there is a finite algebraic field extension $k \subset L$ of degree $n$ with no intermediate fields. For instance, $k \subset L$ could be chosen to be separable of degree $n$ and such that the Galois group of the normal closure of $L$ over $k$ is the symmetric group on $n$ letters; since there are no subgroups properly between the symmetric group on $n$ letters and one of its subgroups of index $n$, i.e., the stabilizing subroup of one of the letters, there are no fields between $k$ and $L$. Let $t$ be an indeterminate over $L$. Then the subring $R$ of the power series ring $L[[t]]$ consisting of those power series with constant term in the smaller field $k$, i.e., $R=k+t L[[t]]$, is a domain with the desired property. In this case, the multiplicity of $R$ is $n$ and if $n>2$, there do exist nonzero ideals of $R$ which are not Ratliff-Rush. For if $x \in L-k$, then $L=k(x)$, and $I=(t, x t) R$ has the maximal ideal of $R$ as its associated Ratliff-Rush ideal, so $x^{2} t \in \widetilde{I}-I$.

(2.11) Suppose $(R, M)$ is a one-dimensional local domain, $I$ is a nonzero proper ideal of $R$, and $S$ is the blowup of $I$. From the result of Lipman mentioned in (1.14), all sufficiently high powers of $I$ are stable. If $I^{n}$ is stable and $a I^{n}=I^{2 n}$, then $S=I^{n} / a$ and the minimal number of generators of $I^{n}$ is the same as the minimal number of generators of $S$ as an $R$-module; by Nakayama's lemma, this is the vector space dimension of $S / M S$ over $R / M$. Therefore, for all sufficiently large $n$, the minimal number of generators of $I^{n}=\widetilde{I^{n}}$ is the cardinality of $S / M S$ as a vector space over $R / M$. We remark that this stable value is always at least as large as the minimal number of generators of $I$. For, the minimal numbers of generators of $I$ and of the 
$R$-module $S$ do not change if we change the base ring $R$ by tensoring with a faithfully flat extension; and as discussed in (3.3) below, there is a faithfully flat extension $R(X)$ of $R$ for which $I R(X)$ has a principal reduction $f R(X)$. Since the blowup $S \otimes_{R} R(X)$ now has the form $R(X)[I R(X) / f]$, it is generated as an $R(X)$-algebra by one fewer than the minimal number of generators of $I$. If $S$ properly contains $R$, i.e., if the ideal $I$ is not principal, then the number of generators for $S$ as an $R$-module is at least one greater than the number of generators of $S$ as an $R$-algebra. (For, since any set of preimages in $S$ of an $R / M$-basis for $S / M S$ is a generating set for $S$ as an $R$-module, 1 is an element of a minimal generating set for $S$.) Therefore the minimal number of generators of $I$ is less than or equal to this stable value of the minimal number of generators of high powers of $I$. As is illustrated in Example 2.12, it can happen that a given element of $I$ is part of a minimal generating set for $I$ but not part of a minimal generating set for $\widetilde{I}$.

Example 2.12. Let $R=k\left[\left[t^{4}, t^{5}, t^{11}\right]\right]$ and $I=\left(t^{8}, t^{9}, t^{15}\right) R$. Then the blowup of $I$ is $k[[t]]$, so $\widetilde{I}=\left(t^{8}, t^{9}, t^{10}, t^{11}\right) R$ is the integral closure of $I$. Thus, $t^{15}$ is part of a minimal generating set for $I$ but is not part of a minimal generating set for $\widetilde{I}$.

General Example 2.13. Let $(R, M)$ be a one-dimensional Gorenstein local domain that is not integrally closed, and let $K$ be its field of fractions. Then

$$
M: K M=\{x \in K: x M \subseteq M\}=\{x \in K: x M \subseteq R\}=M^{-1}
$$

is a ring, the unique minimal overring of $R$ in $K$. Since $R$ is Gorenstein, every nonzero fractional ideal of $R$ is reflexive [B2, Theorem 6.3, page 18], so there are no $R$-modules between $R$ and $M^{-1}$. Thus, the length of the $R$-module $M^{-1} / M$ is 2 , and $M^{-1}$ has at most two maximal ideals. Let $I$ be an ideal in $R$ having blowup $M^{-1}$; then we can choose an element $a \in I$ such that $a M^{-1}=I M^{-1}$. Since the blowup $M^{-1}$ of $I$ properly contains $R, I$ is not principal, so we have $a R<I \subseteq a M^{-1}$, and since there are no $R$-modules between $R$ and $M^{-1}$, there are none between $a R$ and $a M^{-1}$, so $I=a M^{-1}$. Thus, the ideals of $R$ having blowup $M^{-1}$ are precisely the unique minimal overideals of principal ideals, i.e., the ideals of the form $a M^{-1}, a \in M-0$; these ideals are two-generated, and since $\left(a M^{-1}\right)^{2}=a\left(a M^{-1}\right)$, they are stable, their associated graded rings are Cohen-Macaulay, and all their powers are Ratliff-Rush.

For instance, if $R$ is the Gorenstein ring $k\left[\left[t^{4}, t^{5}, t^{6}\right]\right]$ and $I=\left(t^{4}, t^{11}\right) R$, then $I$ is the unique minimal overideal of $t^{4} R$, so $\mathrm{G}(I)$ is Cohen-Macaulay and $I$ and its powers are Ratliff-Rush. 
Example 2.14. We show that every Ratliff-Rush ideal $I$ in $R=k\left[\left[t^{3}, t^{4}\right]\right]$ has the property that its powers are Ratliff-Rush. The unique minimal overring of $R$ is $R\left[t^{5}\right]$ and by General Example 2.13 the ideals of $R$ that have blowup $R\left[t^{5}\right]$ are stable. Since the ring $R\left[t^{5}\right]=k\left[\left[t^{3}, t^{4}, t^{5}\right]\right]$ also has a unique minimal overring $R\left[t^{2}\right]$ inside $k[[t]]$ (even though $R\left[t^{5}\right]$ is not Gorenstein), it remains to examine the Ratliff-Rush ideals with blowup $R\left[t^{2}\right]$ and with blowup $R[t]$. The powers of the maximal ideal $M$ of $R$ are readily seen to be integrally closed and hence Ratliff-Rush. Indeed, although $M$ is not stable, $M^{n}$ is stable for $n \geq 2$, since for any integer $r \geq 6$, the integrally closed ideal $\left(t^{r}, t^{r+1}, t^{r+2}\right) R$ is stable. The only nonzero proper integrally closed ideal whose blowup is not $k[[t]]$ is $\left(t^{4}, t^{6}\right) R$ and this ideal is stable. The Ratliff-Rush ideals having blowup $R\left[t^{2}\right]=k\left[\left[t^{2}, t^{3}\right]\right]=S$ are contractions of principal ideals of $S$. A nonzero proper principal ideal in $S$ is of the form $\left(t^{n}+a t^{n+1}\right) S$ for some integer $n \geq 2$ and $a \in k$. If $n \geq 6$, then the contraction of this principal ideal of $S$ to $R$ is $\left(t^{n}+a t^{n+1}, t^{n+2}\right) R$, and this ideal in $R$ is stable. The contraction to $R$ of $\left(t^{3}+a t^{4}\right) S$ is $\left(t^{3}+a t^{4}, t^{8}+a t^{9}\right) R$; this ideal is stable because its blowup is $R\left[t^{5}\right]$, as noted above. If $a \neq 0$, then $\left(t^{4}+a t^{5}\right) S \cap R=\left(t^{6}, t^{7}, t^{8}\right) R=M^{2}$, a stable ideal, while $t^{4} S \cap R=\left(t^{4}, t^{6}\right) R$ again a stable ideal. Finally, $\left(t^{5}+a t^{6}\right) S \cap R=\left(t^{7}, t^{8}, t^{9}\right) R$, an integrally closed stable ideal in $R$. We conclude that every Ratliff-Rush ideal in $R$ has the property that its powers are again Ratliff-Rush.

\section{Noetherian domains in which every nonzero ideal is Ratliff-}

Rush. Ratliff and Rush [RR, (2.4)] prove that every nonzero ideal in a Dedekind domain is what we are calling a Ratliff-Rush ideal. They also [RR, Remark 2.5] express interest in classifying the Noetherian domains in which every nonzero ideal is a Ratliff-Rush ideal. This interest motivated the next sequence of results. We show first that a domain with this property has dimension at most one.

Proposition 3.1. Let $R$ be a Noetherian domain. If every nonzero ideal in $R$ is Ratliff-Rush, then the dimension of $R$ is at most one.

Proof. Suppose $R$ has dimension at least two; let $(x, y) R$ be an ideal of height two, and set $I=\left(x^{4}, x^{3} y, x y^{3}, y^{4}\right) R$. By [Ku, Chapter V, Theorem 4.14], $\{x, y\}$ is an independent set, so $x^{2} y^{2} \notin I$. But $I^{2}=((x, y) R)^{8}$, so $x^{2} y^{2} \in \widetilde{I}$; i.e., $I$ is not a Ratliff-Rush ideal.

By (1.7) and (1.9), a one-dimensional Noetherian domain $R$ has the property that every nonzero ideal in $R$ is Ratliff-Rush iff for each maximal ideal 
$M$ of $R, R_{M}$ has this property. So in this section we usually assume that the domain is local.

Proposition 3.2. Let $R$ be a one-dimensional local domain. If $R$ has an ideal I some power of which requires a different minimal number of generators than I itself, then $R$ has a nonzero ideal that is not Ratliff-Rush.

Proof. Suppose that $I^{s}$ requires a different number of generators than $I$. Let $S$ be the blowup of $I$ and $a$ be in $S$ such that $I S=a S$. Since $a$ is in the Jacobson radical of $S$, for some large $n, a^{n}$ is in the conductor of $S$ into $R$. Now $a^{n} I^{s}$ and $a^{n+s-1} I$ also have blowup $S$, and they have the same extension to $S$ and hence the same associated Ratliff-Rush ideal $\widetilde{I^{n+s}}$. But since they require different numbers of generators, they are not both equal to $\widetilde{I^{n+s}}$.

(3.3) In Proposition 3.2 we were showing the existence of an ideal that is not Ratliff-Rush. When trying to show that a given ideal in a local domain is Ratliff-Rush, we may assume that the residue field is infinite (and hence that every ideal has a minimal reduction generated by analytically independent elements). This is justified by a standard construction: If $(R, M)$ is a local domain and $X$ is an indeterminate, then $R(X)=R[X]_{M[X]}$ is a faithfully flat extension of $R$ with infinite residue field $(R / M)(X)$. A given ideal in $R$ is Ratliff-Rush iff its extension to $R(X)$ is Ratliff-Rush, by (1.7). Thus, if every nonzero ideal in $R(X)$ is Ratliff-Rush, then the same is true in $R$. We could not apply this construction in the proof of Proposition 3.2 because even if the ideal $I$ of the hypothesis is replaced by $I R(X)$, the ideals $a^{n} I^{s} R(X)$ and $a^{n+s-1} I R(X)$ may not be extended from $R$, so their contractions to $R$ may be Ratliff-Rush even if they are not.

Corollary 3.4. If $R$ is a one-dimensional local domain for which the embedding dimension is less than its multiplicity, then $R$ has a nonzero ideal that is not Ratliff-Rush.

Proof. The embedding dimension of $R$ is the minimal number of generators of its maximal ideal $M$, while the multiplicity of $R$ is the minimal number of generators of high powers of $M$.

(3.5) Let $R$ be a one-dimensional local domain with integral closure $R^{\prime}$, and consider the following conditions on $R$ : (a) $R$ has multiplicity at most two. (b) Every ideal can be generated by two elements. (c) Every nonzero ideal is stable. Sally and Vasconcelos have shown that: 
(a) $\Leftrightarrow$ (b) [SV1, Proposition 2.1];

(b) $\Rightarrow$ (c) [SV2, Proposition 2.6];

and if $R^{\prime}$ is finitely generated over $R$, then (c) $\Rightarrow$ (b) also [SV1, Theorem 2.4];

but $(\mathrm{c}) \nRightarrow(\mathrm{b})[\mathrm{SV} 2$, Example 5.4].

In their example $R$ has multiplicity three, and the completion of $R$ has nonzero nilpotent elements (which is always the case if $R^{\prime}$ is not finitely generated over $R$ ). The implication (a) $\Rightarrow(\mathrm{c})$ and (1.1) above show that:

Corollary 3.6. Let $R$ be a local domain of dimension one and multiplicity at most two. Then every nonzero ideal of $R$ is a Ratliff-Rush ideal.

Example 3.7. Let $R=k\left[\left[t^{2}, t^{5}\right]\right]$; then by Corollary 3.6, every nonzero ideal in $R$ is Ratliff-Rush. But the ideal $I=\left(t^{4}, t^{7}\right) R$ is neither principal nor integrally closed. (The cube of the element $t^{5}$ is in the cube of $I$, but $t^{5} \notin I$.) The existence of such an ideal $I$ in this ring $R$ follows from Theorem 2.8, since the ring $k\left[\left[t^{2}, t^{3}\right]\right]$ is properly between $R$ and $R^{\prime}=k[[t]]$.

(3.8) It can happen that a one-dimensional local domain $(R, M)$ has multiplicity two and yet the integral closure $R^{\prime}$ of $R$ is not a finitely generated $R$-module. An easy way to construct such an example is to begin with the field $k=\mathbb{Z} / 2 \mathbb{Z}$, and choose $y$ in the formal power series ring $k[[x]]$ such that $y$ has positive order and $x$ and $y$ are algebraically independent over $k$. (It is easy to see by a cardinality argument that the transcendence degree of the field of fractions $k((x))$ of $k[[x]]$ over $k$ is infinite and even uncountable.) We have that $k\left(x, y^{2}\right) \subset k(x, y)$ is a purely inseparable field extension of degree two. Let $V=k[[x]] \cap k\left(x, y^{2}\right)$, and $W=k[[x]] \cap k(x, y)$. Then $V \subset W$ are DVR's with $k\left(x, y^{2}\right)$ and $k(x, y)$ as fields of fractions, and $W$ is the integral closure of $V$ in $k(x, y)$. Moreover, the maximal ideal $x V$ of $V$ extends to generate the maximal ideal in $W$, and $V$ and $W$ both have residue field $k$, the residue field of $k[[x]]$. Therefore, $W$ is not a finitely generated $V$-module, for if it were, Nakayama's lemma would imply that $V=W$. Let $R=V[y]$. Then $R$ is a one-dimensional local domain of multiplicity two such that the integral closure $R^{\prime}=W$ of $R$ is not a finitely generated $R$-module. (In this example, the $R$-subalgebras of $R^{\prime}$ are linearly ordered with respect to inclusion [GH, Example 2.22].)

We finish by showing that, in a one-dimensional local domain, the property that every nonzero ideal is Ratliff-Rush implies the apparently stronger property that every nonzero ideal is stable; and we show that these properties impose stringent conditions on a local domain of multiplicity greater 
than two. We are grateful to Roger Wiegand, who showed us reference $[\mathrm{H}]$ and pointed out the necessity of adding the last part to conditions (c) and $(\mathrm{d})$.

Theorem 3.9. Let $(R, M)$ be a one-dimensional local domain, and $R^{\prime}$ be its integral closure. Then the following conditions are equivalent:

(a) Every nonzero ideal in $R$ is stable; i.e. $R$ is a stable domain in the sense of [L], [SV1], [SV2].

(b) Every nonzero ideal in $R$ is Ratliff-Rush.

(c) Every $R$-submodule of $R^{\prime}$ that contains $R$ is a ring, and $R^{\prime} / M R^{\prime}$ is not isomorphic to a direct product of three copies of $\mathbb{Z} / 2 \mathbb{Z}$.

(d) For every $x$ in $R^{\prime}, \operatorname{dim}_{R / M}(R[x] / M R[x]) \leq 2$, for every $x$, $y$ in $R^{\prime}$, $\operatorname{dim}_{R / M}(R[x, y] / M R[x, y]) \leq 3$, and $R^{\prime} / M R^{\prime}$ is not isomorphic to a direct product of three copies of $\mathbb{Z} / 2 \mathbb{Z}$.

Moreover, if these conditions hold and $S$ is any ring between $R$ and its field of fractions, then every nonzero ideal in $S$ is stable and hence Ratliff-Rush.

Proof. (a) $\Rightarrow$ (b) by (1.1).

(b) $\Rightarrow$ (a) : Assume there is a nonzero ideal $I$ in $R$ that is not stable. Then by (1.1), $I$ does not have a principal reduction, i.e., the principal ideal $I R^{\prime}$ is not generated by an element of $I$. By induction on the minimal number of generators of $I$, we can find a two-generated ideal $J$ contained in $I$ that has no principal reduction. Then $J^{2}$ is also two-generated by Proposition 3.2, so by the proof of [SV2, Theorem 3.4], $J$ is stable, a contradiction.

To show that (c) and (d) are equivalent, we prove:

Sublemma 3.9.1. For $(R, M)$ a quasilocal ring and $R^{\prime}$ an integral extension ring of $R$, the following conditions are equivalent:

$\left(c^{\prime}\right)$ Every $R$-submodule of $R^{\prime}$ that contains $R$ is a ring.

$\left(d^{\prime}\right)$ For every $x$ in $R^{\prime}, \operatorname{dim}_{R / M}(R[x] / M R[x]) \leq 2$, and for every $x, y$ in $R^{\prime}, \operatorname{dim}_{R / M}(R[x, y] / M R[x, y]) \leq 3$.

Proof. $\left(\mathrm{c}^{\prime}\right) \Rightarrow\left(\mathrm{d}^{\prime}\right)$ : For $x$ in $R^{\prime}, R+R x=R[x]$; and for $x, y$ in $R^{\prime}$, $R+R x+R y=R[x, y]$.

$\left(\mathrm{d}^{\prime}\right) \Rightarrow\left(\mathrm{c}^{\prime}\right)$ : It suffices to show that, for $x, y \in R^{\prime}, R+R x+R y=R[x, y]$. The first part of condition $\left(\mathrm{d}^{\prime}\right)$ implies that $x^{2} \in R+R x+M R[x]$, so $R[x]=R+R x+M R[x]$, and hence by Nakayama's lemma, $R[x]=R+R x$. Similarly $R[y]=R+R y$. Thus, if $y \in R[x]$ or $x \in R[y]$, then $R+R x+R y=$ $R[x, y]$; so suppose neither of these holds. Then the images of $1, x, y$ in $R[x, y] / M R[x, y]$ are linearly independent over $R / M$, so the second part of 
condition $\left(\mathrm{d}^{\prime}\right)$ and another application of Nakayama's lemma implies that $R[x, y]=R+R x+R y$ in this case.

Now we complete the proof of Theorem 3.9.

(a) $\Rightarrow$ (c) : By [R, Theorem 2.4], (a) implies $\left(\mathrm{c}^{\prime}\right)$ and the condition that $R^{\prime}$ has at most two maximal ideals. It follows from the latter condition that $R^{\prime} / M R^{\prime}$ cannot be isomorphic to $\mathbb{Z} / 2 \mathbb{Z} \times \mathbb{Z} / 2 \mathbb{Z} \times \mathbb{Z} / 2 \mathbb{Z}$.

(c) $\Rightarrow(\mathrm{a})$ : By $\left(\mathrm{c}^{\prime}\right)$ and [H, Lemma 5 , page 146], for any $R$-subalgebra $S$ of $R^{\prime}$ that is finitely generated over $R$, either $S / M S$ has $R / M$-dimension at most two (and hence $S$ has at most two maximal ideals) or $S / M S \cong$ $\mathbb{Z} / 2 \mathbb{Z} \times \mathbb{Z} / 2 \mathbb{Z} \times \mathbb{Z} / 2 \mathbb{Z}$. If the latter alternative were to hold for some $S$, then all the finitely generated $R$-subalgebras $T$ between $S$ and $R^{\prime}$ would have at least three maximal ideals, so that $T / M T \cong \mathbb{Z} / 2 \mathbb{Z} \times \mathbb{Z} / 2 \mathbb{Z} \times \mathbb{Z} / 2 \mathbb{Z}$, and hence $R^{\prime} / M R^{\prime} \cong \mathbb{Z} / 2 \mathbb{Z} \times \mathbb{Z} / 2 \mathbb{Z} \times \mathbb{Z} / 2 \mathbb{Z}$, a contradiction. Hence $R^{\prime}$ has at most two maximal ideals. By [R, Theorem 2.4], $R$ is stable.

Finally, suppose conditions (a)-(d) hold and $J$ is a nonzero ideal in a ring $S$ between $R$ and its field of fractions. If $J=\left(x_{1}, \ldots x_{n}\right) S$, choose a nonzero $a \in R$ so that $a x_{1}, \ldots, a x_{n} \in R$ and let $I=\left(a x_{1}, \ldots, a x_{n}\right) R$. Since every nonzero ideal in $R$ is stable, there exists $b \in I$ such that $b I=I^{2}$. Thus, $b a J=b I S=I^{2} S=a^{2} J^{2}$, and since $b / a \in J$, we see that $J$ is stable.

Example 3.10. Let $F$ be a field of characteristic two, and let $x, y$ be elements of a field extension that are algebraically independent over $F$. Let $R^{\prime}=F(x, y)[[t]]$ and $R=\left\{f(t) \in R^{\prime}: f(0) \in F\left(x^{2}, y^{2}\right)\right\}$. Then by Theorem 3.9, $R$ has ideals that are not Ratliff-Rush. But we contend that every nonzero two-generated ideal in $R$ is stable and hence Ratliff-Rush. For, if the element $f(t)$ of $R$ has order $n$ as a power series in $t$, then $t^{n+1} R^{\prime} \subseteq f(t) R$. Hence if $I$ is a nonzero two-generated ideal in $R$, then $I=(f(t), g(t)) R$ where $f(t), g(t)$ are power series of the same order, say $n$. Let $a, b$ be the leading coefficients (i.e., the coefficients of $t^{n}$ ) in $f(t), g(t)$ respectively; then $a^{2} / b^{2} \in F\left(x^{2}, y^{2}\right)$, so $f^{2}, g^{2}$ are associates in $R$, and hence $I^{2}=\left(f^{2}, f g\right) R=f I$.

Corollary 3.11. Let $(R, M)$ be a one-dimensional local domain with multiplicity $e>2$, and let $R^{\prime}$ be the integral closure of $R$. Assume that the conditions of Theorem 3.9 hold. Then

(1) $R^{\prime}$ is local, i.e., a DVR,

(2) the residue field of $R^{\prime}$ is (isomorphic to) $R / M$ (under the canonical map of $R^{\prime}$ onto its residue field), 
(3) $R^{\prime}$ is not finitely generated as an $R$-algebra, and

(4) for each $R$-subalgebra $S$ of $R^{\prime}$, the square of the (unique) maximal ideal of $S$ is contained in $M S$.

Conversely, if conditions (1), (2) and (4) hold (without hypothesis on the multiplicity), then $R$ is a stable domain.

Proof. Statements (1), (2), and (3) are precisely statements (b), (d), and (a) of Theorem 3.3 of $[\mathrm{R}]$. For (4), we use condition (d) of Theorem 3.9: Denote $R / M$ by $k$, and suppose (4) fails, i.e., the unique maximal ideal $\bar{N}$ in the $k$-algebra $\bar{S}=S / M S=k \oplus \bar{N}$ does not satisfy $\bar{N}^{2}=0$, i.e., there are elements $\bar{x}, \bar{y}$ of $\bar{N}$ for which $\overline{x y} \neq 0$. Now if $\bar{y} \in \bar{x} \bar{S}$, then $\bar{x}^{2} \neq 0$, so a preimage $x$ in $S$ of $\bar{x}$ violates (d); so we may assume that $\bar{y} \notin \bar{x}$ and $\bar{x} \notin \bar{y} \bar{S}$. We show that preimages $x, y$ in $S$ of $\bar{x}, \bar{y}$ violate (d) by showing that $\bar{x}, \bar{y}, \overline{x y}$ are $k$-linearly independent: Suppose $a, b, c$ are elements of $k$ for which $a \bar{x}+b \bar{y}+c \overline{x y}=0$. If $a \neq 0$, then $a+c \bar{y}$ is a unit in $\bar{S}$, so $\bar{x}=-(a+c \bar{y})^{-1} b \bar{y} \bar{S} \in \bar{y} \bar{S}$, a contradiction; and similarly $b=0$, so $c \overline{x y}=0$ and hence $c=0$ also.

Finally, suppose that conditions (1), (2) and (4) hold, and let $x, y \in R^{\prime}$. By (2) we may assume that $x, y$ are in the (unique, by (1)) maximal ideals of $R[x]$ and $R[x, y]$, so all monomials in $x$ or in $x, y$ of degree at least 2 are in $M R[x]$ or $M R[x, y]$. Hence $R[x]=R+R x+M R[x]$ and $R[x, y]=$ $R+R x+R y+M R[x, y]$; so (d) of Theorem 3.9 holds.

(3.12) The example of Sally and Vasconcelos [SV2, Example 5.4] suggests the question: Is there a stable one-dimensional local domain of each (finite) multiplicity? The answer is affirmative: Sally and Vasconcelos need only the construction of Ferrand and Raynaud [FR, Proposition 3.1, as modified in Remarques 3.7 (i)] in which the free module $L$ has rank two; but Ferrand and Raynaud's construction also applies in the case where the free module $L$ is of rank $n$ for any positive integer $n$. This construction yields a one-dimensional local domain $R$ of which the completion $\widehat{R}$ is (isomorphic to) $k\left[\left[x, y_{1}, \ldots, y_{n}\right]\right]$ where $k$ is a field of characteristic two and $\left(\left(y_{1}, \ldots, y_{n}\right) \widehat{R}\right)^{2}=0$. Since $\widehat{R}$ is a stable ring, it follows from (1.7) that $R$ is a stable domain. And the multiplicity of $R$ and $\widehat{R}$ is $n+1$.

(3.13) Under the hypotheses of Corollary 3.11, it follows from (4) that the extension $M R^{\prime}$ of the maximal ideal $M$ of $R$ is either the maximal ideal $M^{\prime}$ of $R^{\prime}$ or $\left(M^{\prime}\right)^{2}$; and in the example of Sally and Vasconcelos $M R^{\prime}=M^{\prime}$. (For a finitely generated $R$-subalgebra $S$ of $R^{\prime}, M S$ cannot be the maximal ideal of $S$ by Nakayama's lemma.) So it seemed natural to ask whether 
it is always true that $M R^{\prime}=M^{\prime}$. Craig Huneke has shown us that the answer is affirmative and kindly agreed to allow us to include his proof for this fact. Suppose that $(R, M)$ is a one-dimensional stable local domain of multiplicity $e>2$, with integral closure $\left(R^{\prime}, M^{\prime}\right)$ and completion $(\widehat{R}, \widehat{M})$. Since $R^{\prime}$ is local but not a finitely generated $R$-module, $\widehat{R}$ has nonzero prime nilradical $N$ [N, Exercise 1, page 122], and every regular ideal in $\widehat{R}$ is stable. Since $\widehat{R} / N$ is complete, its integral closure is local and a finitely generated module over it; and it is a stable domain, so by Corollary 3.11 its multiplicity is at most two. The inclusion of $R$ into $R^{\prime}$ induces a map of $\widehat{R}$ into the completion of $R^{\prime}$ (which is again a DVR) with kernel $N$, and this map is surjective iff $M R^{\prime}=M^{\prime}$, or equivalently iff the multiplicity of $\widehat{R} / N$ is one. The next proposition (applied to $\widehat{R}$ ) shows that the multiplicity cannot be two, so $M R^{\prime}=M^{\prime}$.

Proposition 3.14. (Huneke) Let $(R, M)$ be a one-dimensional CohenMacaulay local ring in which every regular ideal is stable, the nilradical $N$ is prime, the multiplicity of $R / N$ is two, and the integral closure of $R / N$ is local with residue field isomorphic to that of $R$ under the map induced by inclusion. Then $N=0$.

Proof. Assume by way of contradiction that $N \neq 0$. Let $v$ be the valuation (with value group $\mathbb{Z}$ ) associated to the integral closure of $R / N$, and pick $x, y$ in $M$ so that their images $\bar{x}, \bar{y}$ in $R / N$ generate $M / N, v(\bar{x})=2$, and $v(\bar{y})=2 n+1$ is odd. Then $x R$ is a reduction of $M$ (since $M / N$ is integral over $\bar{x}(R / N))$ with reduction number one [H1, Remark (3), page 504]. Note that $\left(x^{n}, y\right) R \cap N \subseteq M N$; for, if $x^{n} a+y b \in N$, then by passing to $R / N$ we see that $a, b \in M$, so $x^{n} a+y b \in M^{2} \cap N \subseteq x R \cap N=x N \subseteq M N$.

Let $z_{1}, \ldots, z_{k}$ be a minimal basis for $N$. Then $x^{n} R$ is a reduction of each of the ideals $\left(x^{n}, y, z_{i}\right) R$ and of $\left(x^{n}, y, N\right) R$ (again because integralness lifts from $R / N)$ with reduction number one. Thus, $y N \subseteq x^{n} R \cap N=$ $x^{n} N$, since $N$ is prime; so there are elements $a_{i j}$ of $R$ for which $y z_{i}=$ $x^{n}\left(\sum_{j} a_{i j} z_{j}\right)$. Since $\left(y-a_{i i} x^{n}\right) z_{i} \in\left(\left(x^{n}, y, z_{i}\right) R\right)^{2}=x^{n}\left(x^{n}, y, z_{i}\right) R$ and $x^{n}$ is a nonzerodivisor, we see that $\sum_{j \neq i} a_{i j} z_{j} \in\left(x^{n}, y, z_{i}\right) R$. We next observe that, for $j \neq i, a_{i j} \in M$ : Assume for example that $a_{i 1} \notin M$. Then $z_{1} \in\left(z_{2}, \ldots, z_{k}\right) R+\left(x^{n}, y\right) R$, and since $\left(x^{n}, y\right) R \cap N \subseteq M N$ as noted above, we have $z_{1} \in\left(z_{2}, \ldots, z_{k}\right) R+M N$, contradicting the minimality of the basis $z_{1}, \ldots, z_{k}$ of $N$.

Now by the determinant trick [LM, Lemma 2.22, page 47], the determinant of the $k \times k$ matrix $y\left(\delta_{i j}\right)-x^{n}\left(a_{i j}\right)$ annihilates $N$, so the fact that 
$R$ is Cohen-Macaulay implies that the image of this matrix over $R / N$ has determinant zero. But an entry on the main diagonal of this matrix has smaller $v$-value than any of the other entries in its row and column, so the product along the main diagonal has strictly smaller $v$-value than any of the other terms in this determinant. Hence this determinant cannot be zero, giving the desired contradiction.

We would like to express our gratitude to Roger Wiegand and to the referee, who informed us of the existence of $[R]$ and whose suggestions greatly improved the paper.

\section{REFERENCES}

[AM] M. F. Atiyah and I. G. MacDonald, Introduction to Commutative Algebra, AddisonWesley, Reading, Mass., 1969.

[B1] Hyman Bass, Torsion free and projective modules, Trans. Amer. Math. Soc. 102 (1962), 319-327.

[B2] Hyman Bass, On the ubiquity of Gorenstein rings, Math. Zeitschr. 82 (1963), 8-28.

[ES] Paul Eakin and Avinash Sathaye, Prestable ideals, J. Algebra 41 (1976), 439-454.

[FR] Daniel Ferrand and Michel Raynaud, Fibres formelles d'un anneau local noethérien, Ann. scient. Éc. Norm. Sup., $4^{e}$ série 3 (1970), 295-311.

[G] Robert Gilmer, Multiplicative Ideal Theory, Marcel Dekker, New York, 1972.

[GH] Robert Gilmer and William Heinzer, On Jónsson algebras over a commutative ring, J. Pure Appl. Algebra 49 (1987), 133-159.

[H] David Handelman, Propinquity of one-dimensional Gorenstein rings, J. Pure Appl. Alg. 24 (1982), 145-150.

[H1] Sam Huckaba, Reduction numbers for ideals of analytic spread one, J. Algebra 108 (1987), 503-512.

[H2] Sam Huckaba, Reduction numbers for ideals of higher analytic spread, Math. Proc. Camb. Phil. Soc. 102 (1987), 49-57.

[Ka] Irving Kaplansky, Commutative Rings, Univ. of Chicago Press, Chicago, 1974.

$[\mathrm{Ku}]$ Ernst Kunz, Introduction to Commutative Algebra and Algebraic Geometry, Birkhäuser, Boston, 1985.

[LM] Max D. Larsen and Paul J. McCarthy, Multiplicative Theory of Ideals, Academic Press, New York and London, 1971.

[L] Joseph Lipman, Stable ideals and Arf rings, Amer. J. Math. 93 (1971), 649-685.

[LS] Joseph Lipman and Avinash Sathaye, Jacobian ideals and a theorem of BriançonSkoda, Mich. Math. J. 28 (1981), 199-222.

[M] Hideyuki Matsumura, Commutative Ring Theory, Cambridge Univ. Press, Cambridge, 1986.

[MR] A. Mirbagheri and L. J. Ratliff, Jr., On the relevant transform and the relevant component of an ideal, J. Algebra 111 (1987), 507-519.

[MRR] Ahmad Mirbagheri, L. J. Ratliff, Jr., and D. E. Rush, Superficial sets of elements for sets of ideals, Comm. Algebra 17 (1989), 3087-3108.

[N] Masayoshi Nagata, Local Rings, Interscience, New York, 1962.

[NR] D. G. Northcott and D. Rees, Reductions of ideals in local rings, Proc. Cambridge Philos. Soc. 50 (1954), 145-158.

[RR] L. J. Ratliff, Jr., and David E. Rush, Two notes on reductions of ideals, Indiana Univ. Math. J. 27 (1978), 929-934.

[R] David E. Rush, Rings with two-generated ideals, J. Pure Appl. Alg., to appear.

[Sh] Kishor Shah, Coefficient ideals, Trans. Amer. Math. Soc., to appear.. 
[SV1] J. Sally and W. Vasoncelos, Stable rings and a problem of Bass, Bull. Amer. Math. Soc. 79 (1973), 574-576.

[SV2] J. Sally and W. Vasconcelos, Stable rings, J. Pure Appl. Algebra 4 (1974), 319336.

[V] Giuseppe Valla, On form rings which are Cohen-Macaulay, J. Algebra 58 (1979), 247-250.

[ZS] Oscar Zariski and Pierre Samuel, Commutative Algebra, vol. II, Springer-Verlag, Berlin, 1975. 